\title{
Pseudohypoaldosteronism type 1 due to novel variants of SCNN1B gene
}

\author{
Yael R Nobel', Maya B Lodish², Margarita Raygada², Jaydira Del Rivero, \\ Fabio R Faucz ${ }^{2}$, Smita B Abraham ${ }^{2}$, Charalampos Lyssikatos ${ }^{2}$, Elena Belyavskaya ${ }^{2}$, \\ Constantine A Stratakis ${ }^{2}$ and Mihail Zilbermint ${ }^{2,4,5}$ \\ ${ }^{1}$ Department of Medicine, Columbia University Medical Center, New York, New York 10032, USA \\ ${ }^{2}$ Section on Endocrinology and Genetics, Program on Developmental Endocrinology and Genetics, Eunice Kennedy \\ Shriver National Institute of Child Health and Human Development, National Institutes of Health, Bethesda, \\ BG 10-CRC, Room 1-3216, 10 Center Drive, Bethesda, Maryland 20814, USA \\ ${ }^{3}$ Medical Oncology Branch, National Cancer Institute, National Institutes of Health, 10 Center Drive, Building 10, \\ Room 12N-226, Bethesda, Maryland, 20892, USA \\ ${ }^{4}$ Division of Endocrinology, Diabetes, and Metabolism, Johns Hopkins University School of Medicine, Baltimore, \\ Maryland 21287, USA \\ ${ }^{5}$ Suburban Hospital, Bethesda, Maryland 20814, USA
}

\author{
Correspondence \\ should be addressed \\ to M Zilbermint \\ Email \\ mihail.zilbermint@nih.gov
}

\section{Summary}

Autosomal recessive pseudohypoaldosteronism type 1 (PHA1) is a rare disorder characterized by sodium wasting, failure to thrive, hyperkalemia, hypovolemia and metabolic acidosis. It is due to mutations in the amiloride-sensitive epithelial sodium channel $(\mathrm{ENaC})$ and is characterized by diminished response to aldosterone. Patients may present with life-threatening hyperkalemia, which must be recognized and appropriately treated. A 32-year-old female was referred to the National Institutes of Health (NIH) for evaluation of hyperkalemia and muscle pain. Her condition started in the second week of life, when she was brought to an outside hospital lethargic and unresponsive. At that time, she was hypovolemic, hyperkalemic and acidotic, and was eventually treated with sodium bicarbonate and potassium chelation. At the time of the presentation to the $\mathrm{NIH}$, her laboratory evaluation revealed serum potassium $5.1 \mathrm{mmol} / \mathrm{l}$ (reference range: $3.4-5.1 \mathrm{mmol} / \mathrm{l}$ ), aldosterone $2800 \mathrm{ng} / \mathrm{dl}$ (reference range: $\leq 21 \mathrm{ng} / \mathrm{dl}$ ) and plasma renin activity $90 \mathrm{ng} / \mathrm{ml} / \mathrm{h}$ (reference range: $0.6-4.3 \mathrm{ng} / \mathrm{ml}$ per h).

Diagnosis of PHA1 was suspected. Sequencing of the SCNN1B gene, which codes for ENaC, revealed that the patient is a compound heterozygote for two novel variants (c.1288delC and c. $1466+1 \mathrm{G}>\mathrm{A}$ ), confirming the suspected diagnosis of PHA1. In conclusion, we report a patient with novel variants of the SCNN1B gene causing PHA1 with persistent, symptomatic hyperkalemia.

\section{Learning points:}

- PHA1 is a rare genetic condition, causing functional abnormalities of the amiloride-sensitive ENaC.

- PHA1 was caused by previously unreported SCNN1B gene mutations (c.1288delC and c.1466+1 G > A).

- Early recognition of this condition and adherence to symptomatic therapy is important, as the electrolyte abnormalities found may lead to severe dehydration, cardiac arrhythmias and even death.

- High doses of sodium polystyrene sulfonate, sodium chloride and sodium bicarbonate are required for symptomatic treatment. 


\section{Background}

Pseudohypoaldosteronism type 1 (PHA1) is a rare genetic condition affecting one in 80000 births (1). It was first described by Cheek \& Perry (2). In affected patients, aldosterone levels are adequate or elevated, but the renal response to aldosterone is disrupted due to functional abnormalities in either the mineralocorticoid receptor (autosomal dominant or sporadic PHA1) or the amiloridesensitive epithelial sodium channel (ENaC) (autosomal recessive PHA1) (Fig. 1).

The ENaC channel is present in all aldosteroneresponsive target organs including kidney, colon, lung and sweat glands. Autosomal recessive PHA1 is associated with permanent resistance to aldosterone in these organs and is clinically characterized by severe sodium wasting, hypovolemia, hyperkalemia, metabolic acidosis and failure to thrive $(3,4)$. Affected patients may also experience recurrent pulmonary infections in a pattern that is indistinguishable from cystic fibrosis (5).

The $\mathrm{ENaC}$ is a trimer composed of three homologous subunits, such as $\alpha-, \beta$ - and $\gamma$ - $\operatorname{ENaC}(6,7)$. Aldosterone resistance may result from mutations in any of these subunit genes: alpha (SCNN1A; 12p13), beta (SCNN1B; $16 \mathrm{p} 12.2-\mathrm{p} 12.1)$ or gamma (SCNN1G; 16p12). As an autosomal recessive disease, both alleles of each gene must be abnormal for this form of PHA1 to occur. Here we present a case of a patient with severe hyperkalemia due to PHA1 resulting from compound heterozygosity for two $S C N N 1 B$ mutations.

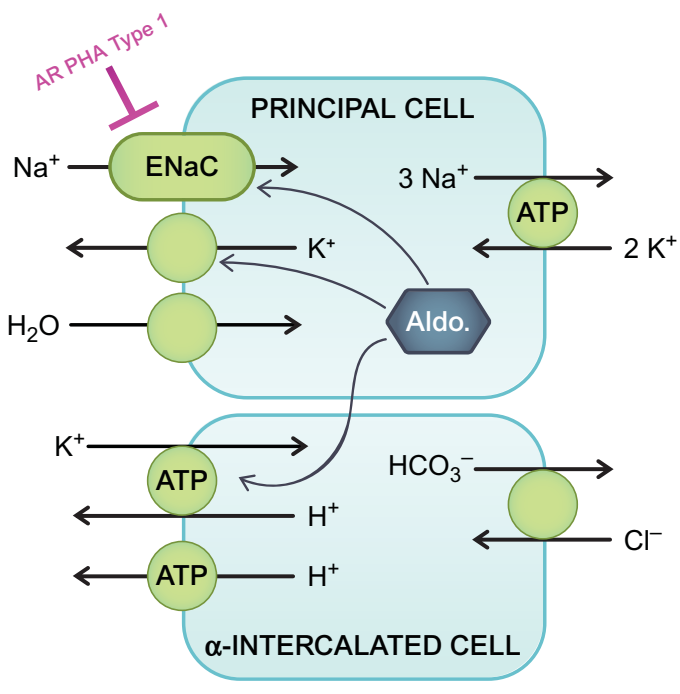

Figure 1

Mechanism of the epithelial sodium channel and disruption by PHA type 1 in the distal nephron. ENaC, epithelial sodium channel; Aldo., aldosterone; AR PHA Type 1, autosomal recessive pseudohypoaldosteronism type 1.

\section{Case presentation}

A 32-year-old African American female was referred to the National Institutes of Health (NIH) Clinical Center with the chief complaint of hyperkalemia and muscle aches.

At 2-3 weeks of age, the patient was found to have multi-organ dysfunction when she was brought to a children's hospital lethargic and unresponsive. At that time, she was found to be hyperkalemic, hypovolemic and acidotic. She was hospitalized multiple times until 2 years of age and maintained on sodium chloride repletion, sodium bicarbonate, potassium chelation and a diet of high salt and limited potassium. She was also prescribed fludrocortisone with no clinical response. After hospitalization, she received sodium chloride, sodium bicarbonate and potassium chelation via gastrostomy tube until being transitioned to the respective oral medications at 3.5 years of age. The patient also reported recurrent episodes of chronic bronchitis during childhood.

She was clinically stable until age 32 years, when she began to experience severe body aches, with pain limiting her motion and no relief to multiple analgesic medications. She also noted heart palpitations and frequent episodes of hidradenitis suppurativa. In September 2012, she was admitted to an outside hospital and found to have a serum potassium level of $6.6 \mathrm{mmol} / 1$ (reference range: $3.5-5.1 \mathrm{mmol} / \mathrm{l}$ ) and heart rate of 150 beats/min. Hyperkalemia was treated with fluid repletion and sodium polystyrene sulfonate, and she was referred to our clinic. The patient's presentation at this age was postulated to be due to medication non-adherence.

\section{Past medical and surgical history}

The patient's past medical history included obesity and a history of pulmonary embolism with secondary pulmonary hypertension. She underwent pulmonary endarterectomy at age 29 years, with subsequent resolution of her pulmonary hypertension. Family history included amyotrophic lateral sclerosis in her father and was negative for other medical conditions including PHA.

\section{Investigation}

\section{Evaluation at the NIH}

On presentation to the NIH, she complained of diffuse muscle aches and increased thirst. Physical exam was notable for blood pressure of $128 / 75 \mathrm{mmHg}$, heart rate 110 beats $/ \mathrm{min}$, BMI $41.4 \mathrm{~kg} / \mathrm{m}^{2}$ and mild acanthosis 
nigricans of the posterior neck. The results of laboratory evaluation are shown in Table 1. Computed tomography scan of the adrenal glands (Fig. 2) showed normal right adrenal gland with no hyperplasia and left adrenal gland with two nodules $1.8 \times 1.5 \mathrm{~cm}$ and $1.6 \times 1.1 \mathrm{~cm}$.

\section{Genetic analysis}

Genetic analysis (performed by GeneDx, Gaithersburg, MD, USA) revealed the presence of two novel heterozygous mutations in the SCNN1B gene (OMIM: 600760; NM_000336.2): c.1288delC, a one-base deletion that generates a frameshift mutation, and c. $1466+1 \mathrm{G}>\mathrm{A}$, an intronic base substitution that leads to a splice site mutation. These variants have not been published in the literature or listed in public domain (http://www.ncbi. nlm.nih.gov/variation/tools/1000genomes/ and http:// exac.broadinstitute.org). Based on these findings, a diagnosis of PHA1 was confirmed. Other candidate genes SCNN1A and SCNN1G were sequenced as well, but no mutations were found.

Table 1 Laboratory evaluation at the National Institutes of Health.

\begin{tabular}{|c|c|c|}
\hline Laboratory test ${ }^{a}$ & Value & $\begin{array}{l}\text { Reference } \\
\text { range }\end{array}$ \\
\hline \multicolumn{3}{|l|}{ Initial presentation } \\
\hline Sodium (mmol/l) & 135 & $136-145$ \\
\hline Potassium (mmol/l) & 5.1 & $3.4-5.1$ \\
\hline 24-h urine sodium ( $\mathrm{mmol} / \mathrm{l})$ & 110 & $40-220$ \\
\hline Bicarbonate $(\mathrm{mmol} / \mathrm{l})$ & 20 & $22-29$ \\
\hline Plasma renin activity ( $\mathrm{ng} / \mathrm{ml}$ per $\mathrm{h}$ ) & 190 & $<0.6-4.3^{b}$ \\
\hline Aldosterone (ng/dl) & 2800 & $<21$ \\
\hline Hemoglobin-A1c (\%) & 5.8 & $<6.5$ \\
\hline \multicolumn{3}{|l|}{ Subsequent evaluation } \\
\hline $\begin{array}{l}\text { Adrenocorticotropic hormone } \\
(\mathrm{pg} / \mathrm{ml})\end{array}$ & 9.6 & $0.0-46.0$ \\
\hline Compound S (ng/dl) & 13.7 & $0.0-49.9$ \\
\hline Insulin-like growth factor 1 (ng/ml) & 76 & $115-307$ \\
\hline Dehydroepiandrosterone (ng/dl) & 155 & $0-599$ \\
\hline $\begin{array}{l}\text { Dehydroepiandrosterone sulfate } \\
(\mathrm{mcg} / \mathrm{ml})\end{array}$ & 0.98 & $0.35-4.30$ \\
\hline Androstenedione (ng/dl) & 93 & $17-175$ \\
\hline 17-OH Progesterone (ng/dl) & 49 & $3-175$ \\
\hline Total testosterone (ng/dl) & $<20.0$ & $<81$ \\
\hline Enhanced estradiol (pg/ml) & 242.7 & $12-460$ \\
\hline Progesterone $(\mathrm{ng} / \mathrm{ml})$ & 1.1 & $0.2-1.5$ \\
\hline Follicle stimulating hormone (U/I) & 4.1 & $3-11$ \\
\hline Luteinizing hormone (U/l) & 5.3 & $1-12$ \\
\hline Prolactin (mcg/l) & 17.4 & $2.0-25.0$ \\
\hline $\begin{array}{l}\text { Sex hormone binding globulin } \\
\text { (nmol/l) }\end{array}$ & 75 & $18-114$ \\
\hline
\end{tabular}

All laboratory tests in serum.

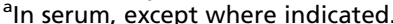

${ }^{b}$ In sodium-replete individuals.

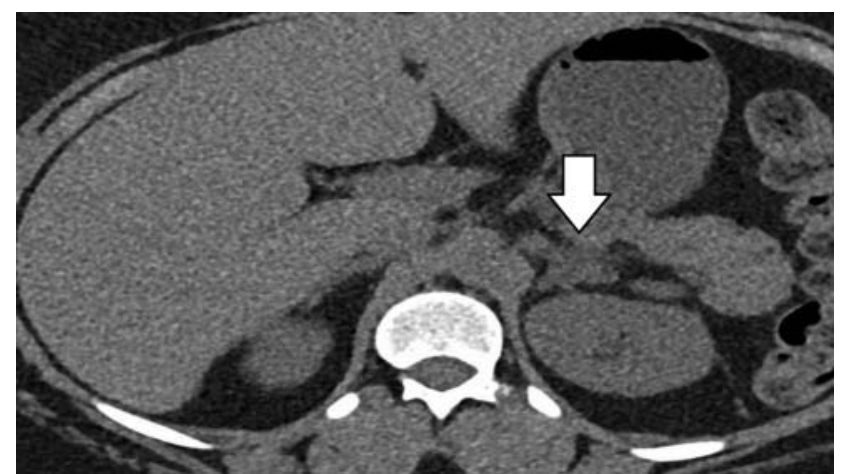

Figure 2

Computed tomography of adrenal glands. Normal right adrenal gland with no hyperplasia and left adrenal gland with two nodules $1.8 \times 1.5 \mathrm{~cm}$ and $1.6 \times 1.1 \mathrm{~cm}$.

In silico analysis was performed in order to better understand the possible protein defects generated by those two mutations. Two different software programs were used: mutation taster (http://www.mutationtaster.org) and Human Splicing Finder (http://www.umd.be/HSF3/ HSF.html). The mutation c.1288delC/p.L430Yfs*3 generated a frameshift mutation probably leading to the loss of the domains: TOPO_DOM Extracellular (amino acids 76-514), TRANSMEM Helical (amino acids 515-545) and TOPO_DOM Cytoplasmic (amino acids 546-640). The mutation c. $1466+1 \mathrm{G}>\mathrm{A}$ probably leads to a splice site donor lost. The predicted new protein, if intron 11 is maintained, is p.R489Rfs ${ }^{\star} 42$. If the splice site donor is lost, the domains that might get lost are the same present in the mutation c.1288delC.

\section{Treatment}

She was treated with sodium polystyrene sulfonate $60 \mathrm{ml} /$ day, sodium chloride $3 \mathrm{G} /$ day and sodium bicarbonate $950 \mathrm{mg} /$ day, which normalized her serum potassium levels, but she continued with diffuse muscle aches.

\section{Outcome and follow-up}

With maintenance sodium polystyrene sulfonate ( $60 \mathrm{ml} /$ day), sodium chloride (3 G/day) and sodium bicarbonate $(950 \mathrm{mg} /$ day) therapy, the patient's electrolyte levels including sodium, potassium and bicarbonate have remained within normal limits. At her most recent evaluation, the patient had been on therapy for 2 years since her initial adult outpatient evaluation at the NIH. Electrolyte levels including sodium, potassium 


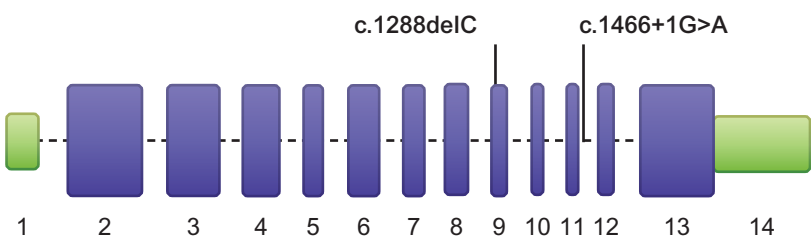

Figure 3

Schematic representation of the SCCN1B gene showing the localization of the two mutations found in the patient. Blue boxes represent the coding exons and green boxes represent the $5^{\prime}$ and $3^{\prime}$ untranslated region (UTR); dotted line represents the intron region.

and bicarbonate were within normal limits. Plasma renin activity and serum aldosterone level remained elevated to $270 \mathrm{ng} / \mathrm{ml}$ per $\mathrm{h}$ (reference range: $\leq 0.6-4.3$ ) and $3500 \mathrm{ng} / \mathrm{dl}$ (reference range: $\leq 26.0$ ) respectively. The patient was tolerating the treatment well. She was counseled regarding the adherence to the treatment regimen, which is crucial in her condition.

\section{Discussion}

We present a case of a patient with two novel heterozygous $S C N N 1 B$ mutations resulting in severe hyperkalemia due to PHA1. Though several cases of patients with PHA1 have previously been presented, the majority of reported mutations are in the subunit of $\alpha-\mathrm{ENaC}$, with mutations in $\beta$ - and $\gamma$-ENaC only rarely described. We speculate that the variants identified are pathogenic because the patient presented with the PHA1 phenotype and the mutations are identified in a gene known to cause PHA1. We also performed an in silico analysis that predicted that both mutations are probably pathogenic. The structure of SCNN1B is shown in Fig. 3. Additional reports of SCNN1B and SCNN1G mutations will help to deepen understanding of different $\mathrm{ENaC}$ subunits' structure, function and contribution to disease in PHA1.

In response to aldosterone, the $\mathrm{ENaC}$ channel is the key mediator of sodium reabsorption from the renal cortical collecting tubule (8). Coupled with the basolateral sodium-potassium ATPase, ENaC acts both directly to reabsorb sodium and indirectly to facilitate potassium secretion (3). As a result, mutations in this channel most commonly produce the clinical picture of failure to thrive, hypovolemia due to sodium wasting, metabolic acidosis and severe hyperkalemia $(3,4)$, as seen in our patient. Although fortunately not present in our patient, severe hyperkalemia may manifest with a range of clinical signs, from weakness or flaccid paralysis (9) to severe arrhythmias (10). PHA1 due to mutations in $\mathrm{ENaC}$ affects a range of organ systems; patients may also present with skin and pulmonary infections or severe diarrhea (11). PHA1 due to mutation in the NR3C2 gene, encoding the mineralocorticoid receptor, is a milder disease affecting only the kidneys, with symptoms generally resolving in infancy (12). In patients suspected of having PHA1 based on these clinical signs and laboratory findings, the diagnosis is formally made through genetic testing to identify mutations in NR3C2, SCNN1A, SCNN1B or SCNN1G.

A limitation of our study is that the patient's parents' genetic information was not obtained. Based on the current understanding of PHA1, we speculate that mutations in the ENaC-coding genes all contribute to autosomal recessive disease, while mutations in mineralocorticoid receptor-coding genes contribute to autosomal-dominant disease. However, without parents' genetic information, we cannot confirm that the mutations identified are autosomal recessive rather than de novo mutations. Future studies should consider assessing genetic information of both patients with PHA1 and their parents in order to better understand the transmission of novel mutations.

Management of patients with PHA1 strives to replace sodium chloride, bicarbonate and fluid, and promote excretion of potassium through administration of high doses of sodium polystyrene sulfonate. In patients with refractory salt wasting, indomethacin may be administered in order to reduce urinary sodium losses through its inhibition of prostaglandin synthesis $(13,14)$.

\section{Declaration of interest}

The authors declare that there is no conflict of interest that could be perceived as prejudicing the impartiality of the research reported.

\section{Funding}

This research work was supported by the Intramural Research Program of the NIH, Eunice Kennedy Shriver National Institute of Child Health and Human Development, protocol 00-CH-0160 (Clinical and Molecular Analysis of ACTH-Independent Steroid Hormone Production in Adrenocortical Tissue). The study had a clinical trial registration number of NCT00005927.

\section{Author contribution statement}

$Y R$ Nobel performed the research for this article and wrote the manuscript. J D Rivero, S B Abraham, C A Stratakis, M B Lodish and M Zilbermint were physicians of the patient. M Raygada provided genetic counseling to the patient. F R Faucz performed in silico analysis and provided assistance with figures. C Lyssikatos and E Belyavskaya coordinated the care of the patient. $M$ Zilbermint coordinated and oversaw the research and publication of the manuscript. 


\section{Acknowledgements}

We thank Diane Cooper, MSLS, National Institutes of Health Library, for providing assistance in writing this manuscript. We thank Nichole Swan and Jeremy Swan, National Institutes of Health Unit on Computer Support Services, for their help in producing and editing figures.

\section{References}

1 Pseudohypoaldosteronism type 1: US National Library of Medicine, National Institutes of Health, Department of Health \& Human Services; 2016 [updated 2016-01-11]. Available from: http://ghr.nlm.nih.gov/ condition/pseudohypoaldosteronism-type-1.

2 Cheek DB \& Perry JW 1958 A salt wasting syndrome in infancy. Archives of Disease in Childhood 33 252-256. (doi:10.1136/adc.33.169.252)

3 Riepe FG 2013 Pseudohypoaldosteronism. Endocrine Development 24 86-95. (doi:10.1159/000342508)

4 Kerem E, Bistritzer T, Hanukoglu A, Hofmann T, Zhou Z, Bennett W, MacLaughlin E, Barker P, Nash M, Quittell L et al. 1999 Pulmonary epithelial sodium-channel dysfunction and excess airway liquid in pseudohypoaldosteronism. New England Journal of Medicine $\mathbf{3 4 1}$ 156-162. (doi:10.1056/NEJM199907153410304)

5 Milla CE \& Zirbes J 2012 Pulmonary complications of endocrine and metabolic disorders. Paediatric Respiratory Reviews 13 23-28. (doi:10.1016/j.prrv.2011.01.004)

6 Canessa CM, Schild L, Buell G, Thorens B, Gautschi I, Horisberger JD \& Rossier BC 1994 Amiloride-sensitive epithelial $\mathrm{Na}+$ channel is made of three homologous subunits. Nature 367 463-467. (doi:10.1038/ 367463a0)

7 Jasti J, Furukawa H, Gonzales EB \& Gouaux E 2007 Structure of acid-sensing ion channel 1 at 1.9 A resolution and low $\mathrm{pH}$.
Nature 449 316-323 http://dx.doi.org/10.1038/nature06163. (doi:10.1038/nature06163)

8 Sauer M, Flemmer A, Thurau K \& Beck F 1990 Sodium entry routes in principal and intercalated cells of the isolated perfused cortical collecting duct. Pflügers Archiv http://link.springer.com/article/10. 1007/BF00370227. Accessed October 15, 2014.

9 Evers S, Engelien A, Karsch V \& Hund M 1998 Secondary hyperkalaemic paralysis. Journal of Neurology, Neurosurgery, and Psychiatry 64 249-252 http://www.ncbi.nlm.nih.gov/pmc/articles/PMC2169962/. (doi:10.1136/jnnp.64.2.249)

10 Mattu AA 2000 Electrocardiographic manifestations of hyperkalemia. American Journal of Emergency Medicine 18 721. (doi:10.1053/ajem.2000. 7344)

11 Rajpoot SK, Maggi C \& Bhangoo A 2014 Pseudohypoaldosteronism in a neonate presenting as life-threatening arrhythmia. Endocrinology, Diabetes \& Metabolism Case Reports 2014 130077. (doi:10.1530/EDM13-0077)

12 Geller DS, Zhang J, Zennaro MC, Vallo-Boado A, Rodriguez-Soriano J, Furu L, Haws R, Metzger D, Botelho B, Karaviti L et al. 2006 Autosomal dominant pseudohypoaldosteronism type 1 : mechanisms, evidence for neonatal lethality, and phenotypic expression in adults. Journal of the American Society of Nephrology 17 1429-1436. (doi:10.1681/ASN. 2005111188)

13 Amin N, Alvi NS, Barth JH, Field HP, Finlay E, Tyerman K, Frazer S, Savill G, Wright NP, Makaya T et al. 2013 Pseudohypoaldosteronism type 1: clinical features and management in infancy. Endocrinology, Diabetes \& Metabolism Case Reports 2013 130010. (doi:10.1530/EDM13-0010)

14 Bommen M \& Brook CG 1982 Pseudohypoaldosteronism: Response to long-term treatment with indomethacin. Archives of Disease in Childhood 57 718-720 http://adc.bmj.com/content/57/9/718.abstract, Accessed March 13, 2015. (doi:10.1136/adc.57.9.718)

Received in final form 4 December 2015 Accepted 7 January 2016 